\title{
Comments
}

THE JOURNAL OF CHEM ICAL PHYSICS

$\operatorname{VOLUME} 5 \mathbf{5}, \mathrm{NUMBER} 7$

1 OCTOBER 1971

\section{On the Unit Cell Group and Factor Group in the Theory of the Electronic and Vibrational Spectra of Crystals*}

\author{
John E. Bertie $\dagger$ \\ Department of Chemistry, The University of Alberta, Edmonton, Canada \\ AND \\ RAOUl KOPELMAN $\ddagger$ \\ Department of Chemistry, The University of Michigan, Ann Arbor, Michigan 48104
}

(Received 2 March 1971)

Bertie and Bell, ${ }^{1}$ in a similarly titled paper, try to clear some literature confusions regarding the unit cell group and the factor group. Their final recommendations as to the proper use of these terms are both correct and consistent with recent usage. ${ }^{2}$ However, their treatment of the alternative definition of the factor group is misleading, and the purpose of this communication is to clarify this point.

Bertie and Bell, enlarging on a brief comment by Kopelman ${ }^{3}$ describe in detail some confusion in papers by Hornig ${ }^{4}$ and Vedder and Hornig, ${ }^{5}$ and propose acceptable 'standard' definitions of the unit cell group and the factor group. However their treatment of Zachariasen's definition of the factor group ${ }^{6}$ fails to do justice to it, and is self-contradicting and misleading. Keeping the terminology of Ref. $1, G$ is the group, $H$ an invariant subgroup, $A^{\prime}, B^{\prime}, C^{\prime}, \cdots$ are coset representatives, so that

$$
G=H+H \cdot A^{\prime}+H \cdot B^{\prime}+H \cdot C^{\prime}+\cdots .
$$

Both Zachariasen and Ref. 1 rewrite this as:

$$
G=H \cdot\left(E^{\prime}+A^{\prime}+B^{\prime}+C^{\prime}+\cdots\right) .
$$

Zachariasen explicitly states that $E^{\prime}$ can be any element of $H$ and defines the bracket in Eq. 2 as the factor group. That the group multiplication is $\bmod H$ is implicit in this definition, otherwise the group would not have the property of closure. Bertie and Bell misquote Zachariasen by defining $E^{\prime}$ as the identity element of both $G$ and $H$ and give the specific application to space group $P 2 / m$ using this definition. They then say that the Zachariasen factor group for this space group can consist of any four elements $\left(E \mid t^{\prime}\right)$, $\left(2 \mid t^{\prime}\right),\left(m \mid t^{\prime}\right),\left(i \mid t^{\prime}\right)$, without reconciling this with their definition of $E^{\prime}$ as the identity operator. In fact Zachariasen's factor group for $P 2 / m$ consists of any four elements $\left(E \mid t_{1}\right),\left(2 \mid t_{2}\right),\left(m \mid t_{3}\right),\left(i \mid t_{4}\right)$, where $t_{1}, t_{2}, t_{3}, t_{4}$, may be the same, all different, all zero, or any combination of these, and the multiplication is $\bmod H$, i.e., mod any combination of primitive translations. If the translational part of the symmetry elements in Zachariasen's factor group is zero or, for nonsymmorphic space groups, the smallest (subprimitive) translation required by the screw axes or glide planes, then his factor group is identical with the unit-cell group specified in Ref. 1. But it is by no means neces- sary that the Zachariasen factor group and this unit cell group be the same, even though the former may still agree with the looser unit cell group definitions of Hornig. ${ }^{4}$

It is implicit in the definition of the unit cell group and Zachariasen's factor group that the multiplication is $\bmod H$. With this qualification, these groups, as well as the accepted factor group, ${ }^{7}$ are simply isomorphic with each other and with the point group of the space group, and with one of the abstract groups. The accepted factor group ${ }^{7}$ has as its elements the cosets, each coset consisting of many elements of the space group. Zachariasen's factor group has as its elements individual elements of the space group. In this sense the two groups differ, in other senses they are equivalent, as already implied by Winston and Halford. ${ }^{8}$

It is noteworthy that the mod $H$ requirement on the multiplication for the Zachariasen factor group and the unit cell group is the very historical reason why the unit cell group could not be simply edfined as the point group of the space group: In nonsymmorphic space groups the point group of the space group ${ }^{9}$ is not a subgroup of the space group while Bertie and Bell can be taken to imply that $\left(E^{\prime}, A^{\prime}, B^{\prime}, C^{\prime}, \cdots\right)$ would always be a subgroup of the space group.

Many of these points were implicit in Ref. 1, but it seems to be desirable to make them explicitly. The points made in this note do not invalidate any of the examples given in Ref. 1, but, we hope, clarify the reasons for their validity. Finally, we like to recommend that, whatever the definition one chooses, this definition should be used consistently and stated explicitly.

\footnotetext{
* This note results from a proposed note by $\mathrm{R}$. Kopelman. To avoid a note plus a response a joint communication was agreed upon.

† Supported by National Research Council of Canada.

$\ddagger$ Supported by NSF grant GP-18718.

${ }^{1}$ J. E. Bertie and J. W. Bell, J. Chem. Phys. 54, 160 (1971).

2 R. Kopelman, J. Chem. Phys. 47, 2631 (1967).

${ }^{3}$ Reference 2 , footnote 5 .

${ }^{4}$ D. F. Hornig, J. Chem. Phys. 16, 1063 (1948).

${ }^{5}$ W. Vedder and D. F. Hornig, Advan. Spectry. 2, 189 (1961).

${ }^{6} \mathrm{~W}$. H. Zachariasen, Theory of X-Ray Diffraction in Crystals (Wiley, New York, 1945), pp. 247-8.

7 E. P. Wigner, Group Theory (Academic Press, New York, 1959), p. 68. The earlier (1931) German edition was historically available to all other authors quoted.

${ }^{8}$ H. Winston and R. S. Halford, J. Chem. Phys. 17, 607 (1949)

- G. F. Koster, Space Groups and their Representations (Academic Press, New York, 1957).
} 